\title{
La réforme éducative au Chili
}

Points de vue et acteurs

\section{Ivan Nuñez}

Traducteur : Claudine Adam

\section{OpenEdition}

Journals

Édition électronique

URL : http://journals.openedition.org/ries/3165

DOI : $10.4000 /$ ries.3165

ISSN : 2261-4265

\section{Éditeur}

Centre international d'études pédagogiques

\section{Édition imprimée}

Date de publication : 1 septembre 1997

Pagination : 47-59

ISSN : 1254-4590

\section{Référence électronique}

Ivan Nuñez, "La réforme éducative au Chili », Revue internationale d'éducation de Sèvres [En ligne],

15 | 1997, mis en ligne le 04 juillet 2013, consulté le 02 mai 2019. URL : http://

journals.openedition.org/ries/3165; DOI : 10.4000/ries.3165

Ce document a été généré automatiquement le 2 mai 2019.

(c) Tous droits réservés 


\title{
La réforme éducative au Chili
}

\section{Points de vue et acteurs}

\author{
Ivan Nuñez
}

Traduction : Claudine Adam

\section{L'enseignement chilien²}

1 Le système éducatif chilien est organisé en :

- niveau préscolaire qui accueille les enfants de moins de 6 ans, dans divers institutions et réseaux publics et privés ;

- niveau de base obligatoire, d'une durée de huit ans dans des écoles municipales ou privées ;

- niveau moyen, de quatre ans dans un lycée avec deux options (scientifique et humaniste, de type général, et technique et professionnelle intégrant études générales et formation professionnelle);

- niveau supérieur, dans des universités et des instituts professionnels ou centres de formation technique.

2 La formation des enseignants est assurée dans les universités et des instituts professionnels. La couverture éducative est acceptable : $97 \%$ des enfants de 6 à 13 ans et $80 \%$ des adolescents de 14 à 17 ans fréquentent les établissements d'enseignement élémentaire et secondaire. L'analphabétisme est de $5 \%$. En moyenne, la population a étudié jusqu'à la neuvième année.

3 La gestion de l'enseignement est mixte : l'État a un rôle cadre, l'enseignement public est décentralisé et la gestion privée importante $(43 \%$ des élèves fréquentent des établissements privés). L'État est chargé de la réglementation, de l'évaluation, il supervise, apporte une aide technique, finance et contrôle.

4 Traditionnellement, c'est le ministère de l'Éducation qui a approuvé les plans et les programmes d'étude nationaux et obligatoires. Mais, en 1990, la nouvelle Loi organique constitutionnelle de l'Éducation a reconnu la compétence des établissements d'enseignement pour élaborer et appliquer leur propre curriculum (décentralisation 
curricula ire). Le ministère de l'Éducation ne s'occupe ni des établissements, ni du personnel enseignant. Pour ce qui est de l'enseignement scolaire, leur gestion est décentralisée dans les municipalités ou les entreprises privées.

Dans l'enseignement supérieur, les universités d'État autonomes et les universités privées ouvertes avant 1980 sont financées par l'État tout comme les universités publiques; les universités, les instituts professionnels et les établissements techniques postsecondaires privés créés après 1980 n'ont pas droit au financement de l'État.

6 Il existe un système de subventions de l'État pour les écoles privées gratuites, valable aussi pour les écoles et les lycées municipaux. Actuellement, $92 \%$ des élèves de l'enseignement élémentaire et de l'enseignement moyen fréquentent les établissements municipaux ou les établissements privés recevant la subvention de l'État. Ce dernier contribue en outre à la décentralisation de l'enseignement en apportant diverses aides techniques et des moyens matériels. Les établissements municipaux, tout comme les établissements privés subventionnés, y ont accès. Depuis 1991, l'État finance les augmentations salariales des enseignants ; salaires et ajustements salariaux sont financés, en grande partie, par la subvention d'éducation qui à cet effet est augmentée proportionnellement.

7 L'État participe aussi au financement de l'enseignement supérieur. Ce dernier est payant pour les étudiants, mais il existe en plus des aides sous forme de crédits et de bourses pour ceux d'entre eux qui ont des ressources plus faibles, ainsi que des fonds pour le développement institutionnel et pour la recherche scientifique et technologique.

Ce qui suit est un résumé du diagnostic réalisé en 1994 par un comité technique formé par des personnalités reconnues du monde intellectuel, éducatif, politique et du monde du travail.

« ...Les progrès obtenus par le pays en ce qui concerne l'accès à l'enseignement et l'augmentation de la durée des années d'études, progrès réalisés au cours des dernières générations sont sans aucun doute substantiels. Ils ont permis une plus grande équité pour entrer dans le système scolaire; l'époque où l'enseignement n'était pas à la portée de tous a pu être dépassée. Pourtant, malgré les progrès obtenus nous ne saurions être satisfaits. Notre système scolaire souffre de graves défauts, il n'est ni de bonne qualité, ni équitable, ni efficace. Les résultats sont médiocres, principalement chez les enfants et les jeunes issus des secteurs les moins favorisés. Bien plus, les progrès obtenus, quelque importance que l'on puisse leur accorder, sont bien peu de choses comparés aux énormes défis qui attendent le pays à l'approche du XXI ${ }^{\mathrm{e}}$ siècle ${ }^{3}$.»

\section{La réforme éducative en marche}

9 Outre un diagnostic, le comité technique dont je viens de parler a fait une proposition de modernisation du système scolaire chilien pour répondre à trois défis : en finir avec l'extrême pauvreté et assurer l'égalité des chances ; faciliter le développement compétitif du pays; moderniser la société chilienne. Il a donc proposé cinq grands objectifs pour transformer le système scolaire :

- offrir à tous un enseignement de qualité ;

- réformer et diversifier l'enseignement de niveau moyen ;

- revaloriser la profession enseignante ;

- accorder plus d'autonomie et de souplesse de gestion et plus d'informations sur les résultats pour avoir des écoles efficaces ; 
- augmenter les investissements éducatifs, tant publics que privés ${ }^{4}$. échelle de valeurs et ses priorités étant différentes, il distingue quatre domaines dans cette opération de la réforme éducative et il réorganise les propositions du comité 5 .

\section{Les programmes pour améliorer la qualité, l'égalité et l'innovation}

11 Ils ont démarré en 1990 sous le premier gouvernement démocratique. Ces programmes sont destinés à atténuer les problèmes les plus aigus du système scolaire et, dans le même temps, à faire naître une dynamique d'innovation pour préparer ou faciliter la réforme ultérieure: distribution gratuite de manuels scolaires, bibliothèques de classe, informatique éducative en réseau, politique de santé, alimentation scolaire, Internet pour les élèves de milieu rural, programme de soutien intensif pour $10 \%$ des écoles ayant obtenu les résultats scolaires les plus bas et programme de soutien intensif aux écoles rurales n'ayant que trois instituteurs ou moins ${ }^{6}$.

On insiste particulièrement sur la « décentralisation pédagogique » qui doit développer les compétences des établissements scolaires et des enseignants pour améliorer les apprentissages et l'innovation pédagogique, par le biais de projets préparés par les établissements et soutenus par le ministère de l'Éducation. En 1996, 3817 «projets d'amélioration éducative » étaient en place dans les écoles primaires, en 1997, ce type de projet a commencé à s'implanter dans les lycées et dans le même temps le nombre total de projets en primaire passait à 5000 .

\section{La rénovation et la décentralisation des programmes}

13 En 1996, fut approuvé le cadre curriculaire commun et obligatoire pour l'enseignement primaire qui est entré en vigueur en $1997^{7}$. Ce cadre est complété par l'«autonomie curriculaire » qui permet à chaque établissement d'élaborer ses propres programmes d'étude, porte ouverte sur un enseignement plus significatif pour l'élève et d'une importance et d'une pertinence sociales et culturelles plus grandes. D'un autre côté, on cherche à sauvegarder l'identité nationale, grâce au programme minimum commun déjà cité.

14 Ce nouveau programme propose d'insister sur l'apprentissage, ciblé sur l'activité des élèves et sur leurs caractéristiques et leurs connaissances préalables. Cela exige le développement de stratégies adaptées à des élèves hétérogènes et oblige à passer d'un enseignement magistral à une pédagogie basée sur l'exploration, la recherche d'informations et la construction de nouvelles connaissances, le tout réalisé par les élèves eux-mêmes. On recherche enfin le développement de savoir-faire et de capacités d'ordre supérieur, grâce à la maîtrise des contenus essentiels.

15 On a aussi défini des « objectifs fondamentaux transversaux » qui doivent contribuer à renforcer la formation éthique de la personne, à orienter le processus de croissance et d'auto-affirmation personnelle, et à orienter la capacité relationnelle de la personne avec autrui et avec le monde ${ }^{8}$. Ces objectifs sont de la responsabilité de tout l'établissement scolaire et doivent être menés à bien grâce à des actions multiples conduites par chaque établissement. 
16 En ce qui concerne l'enseignement moyen, et tout en tenant compte des particularités du niveau secondaire, les bases et la conception des programmes et de la pédagogie sont les mêmes que celles déjà implantées dans le primaire. On envisage une formation commune dans les deux premiers niveaux du lycée (indépendamment du fait que le lycée soit « scientifique et humaniste » ou « technique et professionnel ») qui se prolongera dans les deux niveaux suivants mais qui sera alors associée à une formation différenciée occupant la plus grande partie de l'horaire'.

17 L'implantation du nouveau curriculum doit être achevée en 2001 dans l'enseignement de base et en 2002 dans l'enseignement moyen.

\section{La revalorisation de la profession enseignante}

18 En accord avec les universités du pays, un très gros effort de réforme de la formation initiale des enseignants a été entrepris. De nouvelles possibilités d'évolution professionnelle pour les enseignants en service apparaissent, comprenant: des bourses pour des stages et des diplômes à l'étranger, une offre large de formation continue, l'accès à des moyens tels que l'informatique éducative et plusieurs autres types d'aides. Des possibilités de participation professionnelle sont aussi offertes: élaboration et exécution collective de projets d'amélioration éducative dans les écoles et les lycées, création de groupes professionnels de travail dans tous les lycées et participation aux décisions sur le curriculum de chaque établissement.

19 Enfin, on assiste à un effort soutenu d'augmentation des salaires, qui a permis de doubler leur niveau réel depuis 1990. Le gouvernement estime qu'on est parvenu à une plateforme suffisamment équitable pour pouvoir instaurer des incitations salariales (et symboliques) pour des travaux collectifs de qualité, ce qui a commencé en 1996, avec le versement d'une prime importante à près de 30000 instituteurs et institutrices faisant partie des équipes les plus remarquables de chaque région. Ce mécanisme se répétera tous les deux ans $^{10}$.

\section{Le temps scolaire}

Jusqu'à présent, les écoles et les lycées subventionnés par l'État fonctionnent selon un horaire par demi-journée avec une moyenne annuelle de 800 heures. L'horaire sera étendu à la journée complète à partir de 1997 et cet effort se prolongera jusqu'en 2003. L'augmentation de l'horaire sera réalisée de façon différenciée, avec une moyenne de 200 heures annuelles et progressivement. De la $3^{\mathrm{e}}$ à la $8^{\mathrm{e}}$ année, on parviendra à 1100 heures annuelles et, dans l'enseignement moyen, à 1216 heures annuelles ${ }^{11}$.

21 Le but de cette politique est de créer de meilleures conditions de fonctionnement des établissements pour répondre aux besoins de la réforme éducative. Il est aussi nécessaire de favoriser les familles aux revenus faibles en prolongeant les horaires scolaires et donc la prise en charge des élèves par l'institution, ce qui facilite le travail des mères et réduit le risque social encouru dans la rue par les enfants.

22 Afin d'étendre les horaires, l'État s'engage à financer et à conseiller techniquement les établissements lorsqu'ils adoptent le nouveau régime. Pour couvrir les frais de l'opération, la subvention éducative sera augmentée de $24 \%$ en moyenne. Les 
investissements nécessaires à l'agrandissement ou à l'adaptation de l'infrastructure architecturale des établissements seront eux aussi financés par l'État.

Près de 2900 établissements (en majorité des écoles municipales et surtout rurales) adopteront ce régime à partir de 1997. Une loi qui réglementera et financera l'intégration progressive des établissements ayant besoin d'investissements est en discussion cette année.

\section{La gestion et le financement}

Grâce aux progrès réalisés dans la modernisation de l'administration, qui ont permis d'assouplir la gestion, les municipalités et les directeurs d'établissements municipaux disposent de nouvelles compétences et de nouveaux mécanismes de planification, d'évaluation et d'incitation ont été introduits dans le travail des enseignants. L'investissement public et privé en éducation a aussi été augmenté. Entre 1990 et 1997, le budget de l'Éducation a doublé et la participation des familles a été substantiellement augmentée. Entre 1994 et 1996, l'ensemble des dépenses publiques et privées en matière d'éducation est passé de $4,89 \%$ à $6 \%$ du PIB, pourcentage qui en 1990 n'était que de $4,33 \%$.

\section{Les débats}

Quelques grands points de convergence existent entre les différents acteurs. En même temps, les débats publics sans fin sur l'éducation et les politiques publiques qui s'y rapportent prennent de plus en plus d'importance.

\section{Les principaux points de convergence}

Il existe un vaste consensus entre les principaux acteurs de la vie politique et sociale $\mathrm{du}$ pays, sur les aspects essentiels de la réforme. Deux points font la quasi-unanimité : il est vital pour le développement économique de s'appuyer sur un enseignement différent de celui actuellement pratiqué et l'éducation est un outil capital pour vaincre la pauvreté et améliorer la qualité de vie de la population.

Pour répondre à ce large consensus, le gouvernement a déclaré que la modernisation de l'éducation était sa principale priorité pour la période 1995-2000, il s'est solennellement engagé à accroître les dépenses publiques et privées concernant l'éducation et à les faire passer de $4,5 \%$ à $7 \%$ du PIB dans un délai de huit ans, objectif actuellement en cours de réalisation ${ }^{12}$.

D'autre part, on est aussi tombé d'accord sur une autre préoccupation prioritaire, l'amélioration de la qualité de l'enseignement et l'égalité totale dans sa distribution. Les grands objectifs de la réforme de l'enseignement sont donc largement consensuels. Les divergences s'expriment davantage sur les questions de gestion et de financement que sur les objectifs, les politiques et les programmes purement éducatifs et pédagogiques. 


\section{Les principaux sujets de divergence}

Depuis que la démocratie a été rétablie en 1990, le débat a été très riche. Les questions débattues ont été très diverses. Cet article ne résumera que quatre d'entre elles. Étant donné sa spécificité et sa complexité, il ne sera pas fait état du débat sur l'enseignement supérieur, bien que les contenus des deux premières questions traitées ici couvrent aussi le monde universitaire.

\section{L'institution : État ou marché ? Quelle décentralisation dans l'enseignement?} parce qu'elle est le reflet d'une grande discussion politique sur le rôle de l'État et la place du marché, sur le caractère de la décentralisation de toutes les institutions et, en partie, à cause des problèmes propres au développement de l'enseignement ${ }^{13}$. Sans compter les nuances intermédiaires, on peut distinguer les trois principaux points de vue suivants.

Le point de vue qui met l'accent sur les valeurs de liberté et de compétitivité ; l'éducation formelle est de la responsabilité des familles et des individus dont les relations doivent être régies par les dynamiques du marché. L'État, de façon transitoire et exceptionnelle, a un rôle subsidiaire qui concerne l'éducation des plus pauvres et ce toujours à travers une décentralisation radicale et une réduction de son appareil de gestion éducative ${ }^{14}$. Les propositions spécifiques dérivées de ce point de vue sont :

- la déréglementation de l'éducation au niveau de l'État; la privatisation des institutions éducatives publiques ;

- le financement de l'enseignement obligatoire pour les plus pauvres par le biais de vouchers pour permettre aux familles d'acquérir librement de l'enseignement dans un marché de l'éducation ouvert;

- dans le même temps, l'attribution des sommes correspondant aux actuelles dépenses publiques en matière d'éducation, uniquement sous la forme de subventions et non sous forme d'allocations directes du ministère de l'Éducation.

Les partisans de ce point de vue ont proposé que la réforme soit financée par le produit de la vente des entreprises publiques, y compris les grandes entreprises nationalisées du cuivre, proposition refusée par le gouvernement.

À l'opposé, on insiste sur l'égalité des chances et des parcours d'apprentissage et l'on rappelle le rôle "enseignant» historique de l'État, qui est perçu comme le garant de l'égalité et de l'intégration sociale. Il y a peu de temps encore, on rejetait la décentralisation et on demandait que les écoles publiques soient de nouveau gérées par le ministère de l'Éducation ; on en vient graduellement à accepter une gestion décentralisée de l'enseignement public (bien que pas nécessairement à la charge des municipalités), mais on continue à refuser la loi du marché et on insiste sur la nécessité d'un fort contrôle de l'État dans l'éducation. Ne faisant pas confiance à l'initiative privée, on ne tolère que celle qui est sans but lucratif ${ }^{15}$.

Une troisième position, mixte ou centriste, affirme que le rôle de l'État dans le contrôle et la promotion de l'éducation est nécessaire et qu'il est particulièrement irremplaçable pour obtenir l'égalité sociale. Elle reconnaît en même temps la légitimité de l'activité privée et encourage le dynamisme et l'initiative de la société civile dans le domaine de l'éducation. Cette opinion accepte les grands principes de l'architecture de la 
décentralisation des années quatre-vingt. Mais elle soutient aussi l'ensemble des réajustements des années quatre-vingt-dix, destinés à avancer sur le chemin de l'égalité des chances et de l'amélioration de la qualité et de l'innovation, grâce à des stratégies d'action publique et à des instruments de compétitivité contrôlée, des cadres normatifs souples de l'État, des aides de la gestion publique et des incitations à l'initiative et au renforcement des autonomies et de la solidarité sociale ${ }^{16}$.

\section{Compétitivité ou citoyenneté ? Formation humaine ou formation professionnelle ?}

La fonction du système éducatif dans la modernisation du pays est aussi un thème de débat. Ce sujet, très discuté déjà dans l'histoire, a été de nouveau abordé lorsque la CEPAL et l'UNESCO, deux organismes des Nations-Unies présents à Santiago, ont essayé de concilier formation à la compétitivité internationale et formation à la citoyenneté. Ils ont affirmé que si un effort réel en matière de compétitivité était nécessaire pour que la citoyenneté puisse exister totalement, la compétitivité ne pouvait se maintenir si l'on prenait un retard trop important dans le domaine de la citoyenneté ${ }^{17}$.

Plusieurs voix se sont dès lors élevées pour insister de façon implicite ou explicite sur l'une ou l'autre de ces deux fonctions, tandis que d'autres voix soutiennent l'intégration ou l'articulation équilibrée entre les deux. Bien que personne n'ose refuser formellement l'objectif de formation à la citoyenneté, quelques groupes privilégient l'éducation à la compétition. D'autres, au contraire, refusent la formation à la compétitivité, interprétée comme contraire à l'éthique de solidarité sociale et d'intégration dont l'éducation moderne devrait jeter les bases. Le célèbre biologiste et philosophe Humberto Maturana, par exemple, a proposé la distinction entre «formation humaine» et «formation professionnelle ». Basant les deux définitions sur la «biologie de l'amour », Maturana voit dans la formation humaine la tâche fondamentale de l'éducation scolaire. Il reconnaît la nécessité de la formation professionnelle mais soutient que la promotion de la compétence réduit les possibilités de plein développement des aptitudes pratiques ${ }^{18}$.

\section{Le travail des enseignants}

Il y a un débat sur la fonction enseignante. Les partisans d'une vision moderniste du marché de l'éducation ont défini deux voies pour ajuster la fonction enseignante aux besoins du système qui se transforme :

- la déréglementation de cette fonction réintroduite sur le marché du travail, comme a voulu le faire la dictature dans les années quatre-vingt. Ils ont réclamé la suppression du statut des professionnels de l'éducation, approuvé en 1991 ;

- la reconversion d'une grande partie des enseignants en patrons de petites entreprises collectives que seraient devenus les établissements scolaires publics, passant ainsi des mains des municipalités aux mains de leurs enseignants respectifs ${ }^{19}$.

À l'opposé, on affirme la nécessité de réglementations spécifiques pour protéger le travail enseignant et rendre aux enseignants du secteur municipal leur statut de «service public » qu'ils ont perdu dans les années quatre-vingt. La demande de stabilité d'emploi est très forte, en raison du traumatisme consécutif à la législation de la dictature qui, dans ces mêmes années, a placé les enseignants dans une situation d'extrême instabilité.

En ce qui concerne les salaires des enseignants, on a reconnu de façon unanime leur insuffisance, bien que le gouvernement et ses partisans insistent sur le fait que durant les 
six dernières années des progrès significatifs et répétés aient été réalisés pour remédier au problème et que l'on continuera à avancer jusqu'à ce qu'il soit totalement résolu.

Au-delà des divergences sur le montant des salaires, on discute du caractère des rémunérations. Les partisans de la privatisation critiquent le système légal qui favorise seulement l'ancienneté des enseignants dans leurs postes de travail et réclament par contre la mise en place de primes à la "productivité ${ }^{20} »$ ou, dans une perspective moins radicale, à l'« excellence de l'exercice professionnel » mesuré en termes de résultats dans l'acquisition des apprentissages.

41 À l'autre extrême, on refuse de privilégier les primes au rendement et l'on défend un système de rémunérations égalitaire, différencié uniquement par l'ancienneté, la formation continue ou par les conditions d'exercice, afin de compenser les situations les plus difficiles.

Une troisième position croit nécessaire de combiner, d'une part, l'augmentation d'une rémunération de base commune suffisante pour compenser le déficit historique et, d'autre part, une politique complémentaire de primes pour un travail de grande qualité, qui seraient attribuées à des groupes d'enseignants et non à chacun en particulier, étant donné les difficultés de l'évaluation individuelle du travail et le caractère de plus en plus collectif que l'on reconnait à la fonction enseignante.

Enfin, le débat est vu sous un autre angle: la définition du rôle de l'enseignant. Les secteurs scolaires ont proposé la définition de l'acte d'enseigner comme une " profession » ce qui a été repris par le statut et par la réforme éducative. On insiste sur la nécessité d'avoir une formation universitaire authentique et actualisée, une autonomie professionnelle et des responsabilités dans le travail, enfin, une insertion institutionnelle souple. Dans les faits, la professionnalisation de l'activité enseignante est discutée sous deux angles: le partisan de la privatisation, qui voit les enseignants comme des techniciens salariés de l'entreprise éducative dont la gestion et l'exercice doivent être conformes aux paramètres du marché; le syndicaliste, qui regrette l'appartenance des enseignants à la structure administrative de l'État, ce qui implique aussi le retour à une définition technique du travail enseignant.

\section{Le conflit culturel et ses répercussions sur l'éducation : libéralisme et conservatisme}

$\mathrm{Au}$ cours de ces dernières années, un débat culturel très large s'est ouvert dans le pays. Revêtant de multiples formes et faisant face à une très grande variété de situations, de cas et de problèmes, ce débat oppose positions libérales ou "progressistes" et positions conservatrices. Voici quelques-uns des sujets spécifiques: le divorce et le caractère de l'institution familiale, la liberté d'information et d'expression, menacée par diverses restrictions, le moyen de combattre le SIDA, les comportements des jeunes, les discriminations sexuelles et ethniques, etc.

Les choses se compliquent selon le rôle que les acteurs assignent à l'État, aux Églises, aux moyens de communication et selon la définition des sphères privées et publiques.

Ces conflits commencent à avoir un impact sur l'éducation, sur la participation des acteurs dans ce domaine et sur les conceptions des programmes et de la pédagogie. Tant dans la société que dans le système éducatif, on assiste à une survivance de la culture autoritaire, mais on voit apparaître une dynamique qui commence à la remettre en 
question. Les jeunes demandent qu'on les écoute et qu'on les comprenne, ils réclament un enseignement plus actualisé et plus en rapport avec les problèmes de la vie.

L'éducation sexuelle est aussi un sujet très discuté. Il y a accord sur sa nécessité, mais on diffère énormément sur les responsabilités et les compétences et sur les moyens à mettre en œuvre pour la rendre effective ${ }^{21}$.

On a d'autre part entamé une discussion sur la pédagogie ; elle commence à dépasser les milieux académiques et professionnels et à descendre sur la scène publique. Dans ce dernier cas, ce sont surtout les conceptions cognitives et constructivistes, favorisées par les objectifs de la réforme, qui ont été diffusées, mais certains secteurs culturellement influents commencent à affirmer la nécessité d'une pédagogie traditionnelle, directive, qui insiste sur la transmission des connaissances ${ }^{22}$. Derrière ces divergences, il semble que l'on retrouve les positions conservatrices et libérales.

\section{Les acteurs les plus importants face au débat}

\section{Les principaux protagonistes ${ }^{23}$}

C'est le gouvernement qui a été, traditionnellement, le principal acteur du débat et des décisions concernant l'éducation. Les deux gouvernements démocratiques ont défendu des positions centristes dans les débats sur le fonctionnement institutionnel et ont adopté une vision progressiste dans le domaine culturel. Bien que de nombreux enseignants aient participé aux travaux de la réforme $\mathrm{e}^{24}$, leur syndicat a constitué un pôle conservateur dans le débat sur l'organisation et la gestion; ce n'est que depuis peu qu'il commence à dépasser la question salariale pour penser aux disciplines pédagogiques et au caractère de la profession en elle-même ${ }^{25}$.

Les municipalités, formant au niveau national un important groupe de pression, demandent une plus grande autonomie dans la gestion de leurs écoles et critiquent la façon dont le gouvernement finance l'enseignement public.

51 L'Église catholique, pour sa part, a encouragé la décentralisation, régime qui garantit l'autonomie à son important réseau d'établissements scolaires et, avec les laïcs qui possèdent ou dirigent des écoles privées, elle réclame un meilleur financement de l'État ${ }^{26}$. Elle fait preuve d'autre part d'un conservatisme culturel modéré.

52 Finalement, les chefs d'entreprise sont des acteurs relativement nouveaux sur la scène éducative. Les plus modernes d'entre eux se sont engagés dans la gestion de lycées techniques et demandent une modernisation urgente et radicale de l'enseignement allant vers la privatisation.

\section{Les acteurs secondaires}

Les partis politiques n'inscrivent pas souvent les questions éducatives à leur ordre du jour. Dans ces débats, les plus habiles sont les partis de droite qui soutiennent les positions néolibérales et néoconservatrices; ils sont alliés aux secteurs patronaux et trouvent un écho important dans les médias qui suivent les mêmes orientations. La gauche extra-parlementaire défend une position étatiste qu'elle soutient ou favorise à l'intérieur du syndicalisme enseignant. 


\section{NOTES}

2. Organización de Estados Iberoamericanos para la Educación, la Ciencia y la Cultura, OEI, y Ministerio de Educaciôn de Chile, Sistemas Educatives Nationales, Chile, Madrid, 1993 (édition actualisée en préparation).

3. Comité Técnico Asesor para la Modernización de la Educación, Los desafíos de la educación chilena trente al sigto XXI, Santiago, Editorial Universitaria, 1995.

4. Comité Técnico Asesor..., op. cit., p. 62-76.

5. José Pablo Arellano, Discurso del Ministre de Educación en ceremonia de inauguration del año escolar 1997, Santiago, Ministerio de Educación, 1997 ; «El sentido de la reforma educacional », Revista de Educación, n² 242, mars 1997, p. 24-39 ; Ministerio de Educación, La Reforma en Marcha. Jornada Completa Diurna parajodos, Santiago, 1996.

6. Eduardo Frei R.T., Mensaje Presidencial 21 de mayo de 1996-21 de mayo de 1997, Santiago, Secretaria de Comunicación y Cultura, 1997, p. 209-211.

7. Ministerio de Educación, Objetivos Fundamentales y Contenidos Minimos Obligatorios de la Educación Básica Chilena, Santiago, enero de 1996.

8. Ministerio de Educación (1996), op. cit., p. 10-12.

9. Ministerio de Educación, Objetivos Fundamentales y Contenidos Mínimos Obligatorios de la Educación Media, Santiago, Consulta Nacional para el Nuevo Curriculo de la Educación Media, 1997.

10. Eduardo Frei R.T., op. cit. p. IX-X.

11. Voir note 4.

Revue internationale d'éducation de Sèvres, 15 | 2013 
12. Eduardo Aninat, « Texto del discurso del Ministro de Hacienda », El Mercurlo, Santiago, 30 août 1994, p. C-4.

13. Iván Núñez, « Chile, el camino de la transición », Revista Iberoamericana de Educación, $n^{\circ} 1$, janvavril de 1993, Número monográfico «El Estado y la Educación », p. 130-137 ; FLACSO, Facultad Latinoamericana de Ciencias Sociales, «El Estado, el mercado y la educación en los 90 », in: ENERSIS, Educación en Chile : un desafio de calidad, Santiago, ENERSIS, S.A., 1996, p. 11-55 ; « Estado y educación: una nueva geografia ", Revista de Educación, n²30, Santiago, novembre 1995, p. 24-33.

14. Antonio Sancho, "En busca de un cambio estructural del sistema educativo ", Revista " Empresas_COPEC», Año 10, n 34, Santiago, décembre 1996, p. 2-5 ; Carlos Neely, «Reformas mal planteadas ", El Mercurio, Santiago, 5 mars 1997, p. 2

15. Colegio de Profesores de Chile, A.G., Chile educa a Chile, Documenta base de discusión. Primer Congreso National de Educaciôn, Santiago, 1997.

16. Cristián Cox, La reforma de la educación chilena : contexto, diseño, implementación, Santiago, Taller de Politicas Públicas de CIEPLAN, enero de 1997.

17. CEPAL-UNESCO, Educación y conocimiento: eje de la transformation productiva con equidad, Santiago de Chile, Comisión Económica para America Latina y Oficina Regional de Educaciôn para América Latina y el Caribe de UNESCO, 1992.

18. Humberto Maturana, «Educación y mercado, respuesta a Carlos Neely », Revista de Education, $\mathrm{n}^{\circ} 232$, Santiago, mars 1996, p. 41-46.

19. Joaquin Lavin, «Traspaso de colegios municipales a sociedades de profesores : una alternativa a evaluar ", in : Rolando Franco et al., Viabilidad pólitica e institucional de la reforma educativa en Chile, Santiago, Comisión Econômica para América Latina,-CEPAL, Série Políticas Sociales $n^{\circ} 11$, décembre 1995, p. 45-49.

20. Andrés Allamand, «Respuesta al Ministro de Educación », El Mercurio, Santiago, 28 de enero de 1997, p. E. 9-10.

21. Ministerio de Educación, Chile, Politicas de educación en sexualidad, Santiago, 1993, «Educación sexual : un diálogo necesario ", Revista de Educación n² 239, Santiago, octubre de 1996, p. 24-33 ; P. Jaime Fernândez M., Jornadas de Conversación sobre Afectividad y Sexualidad, JOCAS, la Punta de un Iceberg, Santiago, Fundaciôn Profamilia y Editorial Patris, 1996.

22. «El dilema de aprender a ensehar », El Mercurio, Santiago, 4 mai 1997, Cuerpo E, «Artes y Letras », p. 6-9.

23. Ivan Núñez, "Gobierno, municipalidades y profesorado : dificultades para un consenso », in : Rolando Franco et. al, op. cit., p. 35-37 ; « La Reforma Educativa en el Debate », Revista de Educación, n²29, Santiago, octobre 1995, p. 24-32.

24. «Encuesta nacional: la opinion de los profesores », Revista de Educación, $n^{\circ} 240$, novembre 1996, p. 24-33 ; Osvaldo Verdugo, «Hacia un proyecto nacional de educación equitativa », in : Rolando Franco et al., op. cit., p. 53-59.

25. Jorge Pavez, Comentarios del Colegio de Profesores de Chile, Santiago, Ponencia en el Seminario «Innovar en la ensenanza media : un desafio estratégico », mai 1997.

26. Hector Vargas sdb., Comentarios y sugerencias de la EIDE al nuevo marco curricular de la ensenanza media, Santiago, Ponencia en el Seminario «Innovar en la ensenanza media: un desafio estratégico », mai 1997.

27. P. Jaime Fernández, op. cit. 


\section{RÉSUMÉS}

Le système éducatif chilien, en pleine modernisation, est l'objet de nombreux débats entre les acteurs : État, Église, partis politiques, syndicats et patronats. Si les grands objectifs de la réforme de l'enseignement - amélioration de la qualité de l'enseignement, égalité pour tous, revalorisation de la profession enseignante, notamment - sont largement consensuels, il n'en est pas de même pour les modalités de gestion et de financement (décentralisation, privatisation, statut des enseignants).

INDEX

Mots-clés : politique éducative, réforme de l'enseignement, système éducatif Index géographique : Chili

\section{AUTEURS}

\section{IVAN NUÑEZ}

Conseiller du ministre de l'Éducation, Santiago, Chili 